\title{
Absorbance properties of gold coated fiber Bragg grating sensor for aqueous ethanol
}

\author{
P. T. Arasu \\ punitha@unikl.edu.my
}

\section{A. S. M. Noor}

\section{A. A. Shabaneh}

\section{S. H. Girei}

\section{A. Mahdi}

\section{H. N. Lim}

\section{H. A. Abdul Rashid}

\section{H. Yaacob}

Wireless and Photonics Network Research Centre of Excellence (WiPNET), Faculty of Engineering, Universiti Putra Malaysia, Malaysia

Communication Technology Section, Universiti Kuala Lumpur, Malaysia

Wireless and Photonics Network Research Centre of Excellence (WiPNET), Faculty of Engineering, Universiti Putra Malaysia, Malaysia

Department of Computer and Communication Systems Engineering, Faculty of Engineering, Universiti Putra Malaysia, Malaysia

Wireless and Photonics Network Research Centre of Excellence (WiPNET), Faculty of Engineering, Universiti Putra Malaysia, Malaysia

Wireless and Photonics Network Research Centre of Excellence (WiPNET), Faculty of Engineering, Universiti Putra Malaysia, Malaysia

Wireless and Photonics Network Research Centre of Excellence (WiPNET), Faculty of Engineering, Universiti Putra Malaysia, Malaysia

Department of Computer and Communication Systems Engineering, Faculty of Engineering, Universiti Putra Malaysia, Malaysia

Department of Chemistry, Faculty of Science, Universiti Putra Malaysia, Malaysia

Faculty of Engineering, Multimedia University, Malaysia

Wireless and Photonics Network Research Centre of Excellence (WiPNET), Faculty of Engineering, Universiti Putra Malaysia, Malaysia

Department of Computer and Communication Systems Engineering, Faculty of Engineering, Universiti Putra Malaysia, Malaysia

Optical Fiber Bragg Grating (FBC) is commonly deployed as a wavelength selective filter in telecommunication as well as to detect physical changes such as pressure, temperature and strain in sensing applications. This paper presents an investigation of FBC as a chemical sensor towards ethanol in aqueous solution. Telecommunication standard single mode FBCs were coated with different thicknesses of thin gold films via sputtering deposition method. The combination of Bragg gratings and gold film enhances the evanescent wave on the surface of the optical fiber. It was found that the FBC coated with $50 \mathrm{~nm}$ gold layer exhibits the strongest response towards water with varying concentrations of ethanol. The sensor shows $55 \%$ change in absorbance levels when the concentration of ethanol is increased from 0 to $99.7 \%$ in water.

[DOI: http://dx.doi.org/10.2971/jeos.2014.14018]

Keywords: Optical fiber, Bragg grating, sensor, absorbance, ethanol, gold

\section{INTRODUCTION}

Fiber optic based sensors are rapidly gaining popularity due to their many advantages such as miniaturization, flexibility and immunity to electromagnetic interference and have the capability for remote sensing [1]-[5]. In telecommunication sectors, Fiber Bragg Gratings (FBGs) are commonly used as wavelength selective filters that are created by a periodic modification (grating) of the refractive index at the core resulting in a periodic modulation of the effective index of the waveguide [6]-[8]. The grating period determines the Bragg wavelength, which is the reflected wavelength in the fiber core.

Among various fiber optic sensors configurations, Fiber Bragg
Grating (FBG) based sensors are one of the most popular and widely used within the photonics community. It has great potential for mechanical [9]-[11], chemical [12]-[14] as well as environmental $[15,16]$ sensing applications.

In recent years, studies are being conducted to utilise FBGs as biochemical sensors. It is possible to observe the absorbance properties of a sensing medium via its interaction with the evanescent wave surrounding the grating region. FBG evanescent wave sensors may potentially become valuable technological platforms for chemical and biological sensing applications. Few reports have been made on FBGs as chemical sen- 


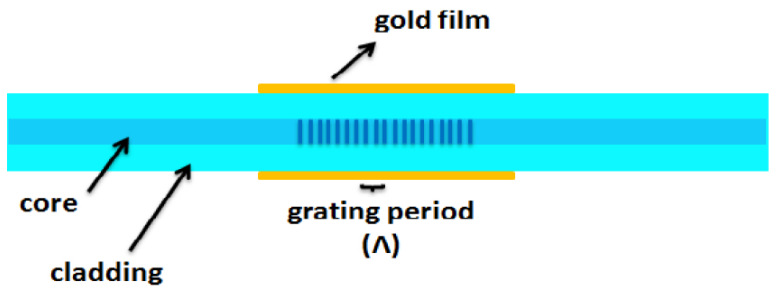

FIG. 1 Schematic diagram of the FBG sensor.

sors by modifying either their structure, such as removal of the cladding $[13,15,17]$ or by modifying the grating formation $[18,19]$.

FBGs are also being deployed as Surface Plasmon resonance sensors. Uniform FBGs [20] and Tilted FBGs [21, 22] give good response towards refractive index changes in the surrounding medium.

In this paper, we compare the absorbance responses of a standard single mode fiber (SMF) and FBG. Both SMF and FBG were prepared with and without gold coatings to investigate their sensitivity and responses towards ethanol. Gold was chosen due to its chemical stability and was sputtered around the SMF and FBG region without removing the fiber optic cladding. The sensor was then tested with different concentrations of ethanol in water to determine it sensing capability.

\section{FIBER BRAGG GRATING SENSOR}

The FBGs used in this research were single mode FBGs with uniform gratings. The polymer coating over the grating area was removed to expose the cladding. A thin gold film is then sputtered over the grating region as shown in Figure 1.

When used with a broadband white light source, Bragg scattering occurs at the grating region and light is scattered in the cladding, creating an evanescent field at the interface between the cladding and the surrounding medium. The gold layer further enhances the evanescent wave [23]. When the fiber sensor is exposed to an analyte surrounding the sensing area, absorption occurs at specific wavelengths corresponding to the analyte's absorbing wavelength. The absorbance coefficient, $\alpha$ is given in Eq. (1) [24].

$$
\alpha=\frac{c \mathcal{E}}{\log _{10} e}
$$

The molar absorptivity is given by $\varepsilon$ and the molar concentration is c. Absorbance (A) is affected by both concentration (c) of the surrounding medium and the path length (l) and is represented by Eq. (2).

$$
\mathrm{A}=\varepsilon .1 . \mathrm{c}
$$

Where $\varepsilon$ is the proportionality constant called the molar extinction coefficient and has the unit liter/mol. It is a constant for a given substance, provided the temperature and wavelength are constant. In this experiment, the absorbance, A, was measured using a spectrophotometer with a visible-NIR light source. The spectrophotometer measured the initial intensity,

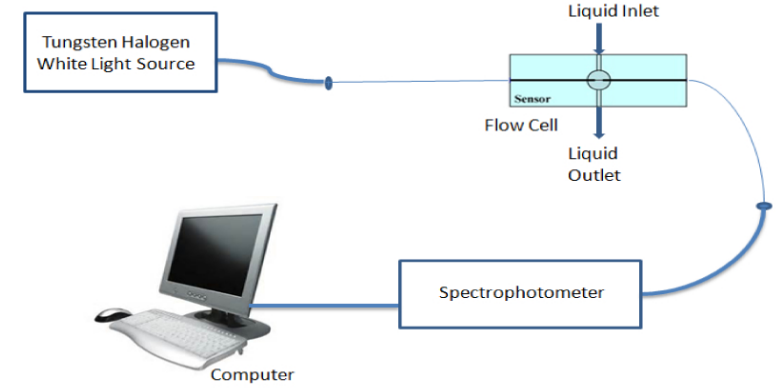

FIG. 2 Experimental setup of the FBG sensor system.

$\mathrm{P}_{0}$, from the uncoated fiber and the sample intensity, $\mathrm{P}$, from the gold coated fiber. Absorbance is the negative logarithm of the transmittance, and absorbance and transmittance carry an inverse relationship as shown in Eq. (3).

$$
\mathrm{A}=-\log \mathrm{T}=-\log \frac{\mathrm{P}}{\mathrm{P}_{\mathrm{o}}}
$$

Using Eq. (3), the absorbance of the developed fiber sensor can be determined by the spectrophotometer software system. The sensing results are based on this measurement.

\section{SENSOR FABRICATION AND EXPERIMENTAL SETUP}

Commercially purchased single mode FBGs with uniform grating periods were used in this research. The grating period of these FBGs are at the telecommunications region at $1550 \mathrm{~nm}$. The gratings are only used for scattering of light to the cladding as the sensor is interrogated at the visible region. The period of the grating does not affect the outcome of the investigation. The buffer coating at the grating area, about $1 \mathrm{~cm}$ long, was removed using $48 \%$ hydrochloric acid $(\mathrm{HCl})$. This was done by immersing the grating region of the FBG into a beaker containing $\mathrm{HCl}$ for 20 minutes. Then the fiber was removed from the acid and washed using distilled water for 2 minutes. After this process, the buffer coating was stripped off manually.

The uncoated FBG was then cleaned with acetone and placed in an EMITECH K575X sputter coater from Quorum Technologies. Gold was sputtered onto the exposed part of the fiber with 0.1 mbar argon gas pressure and sputter current, $50 \mathrm{~mA}$ with a target to sample spacing of $30 \mathrm{~mm}$. The fiber optic was rotated $180^{\circ}$ in order to coat both sides of the fiber. Sputtering time was varied according to the required gold thickness. The same process was also applied for the standard SMF for the purpose of comparison.

The experimental setup for the fiber based sensor is shown in Figure 2. A halogen white light source, HL2000 with a wavelength range between $360 \mathrm{~nm}$ and $1700 \mathrm{~nm}$, from Ocean Optics is used as the input to the sensor. The fiber sensor was placed in a flow cell fabricated using a polyethylene container to minimise evaporation. The output of the fiber sensor was connected to a spectrophotometer, USB4000 with a detector range between $200 \mathrm{~nm}$ and $1100 \mathrm{~nm}$, from Ocean Optics, and 


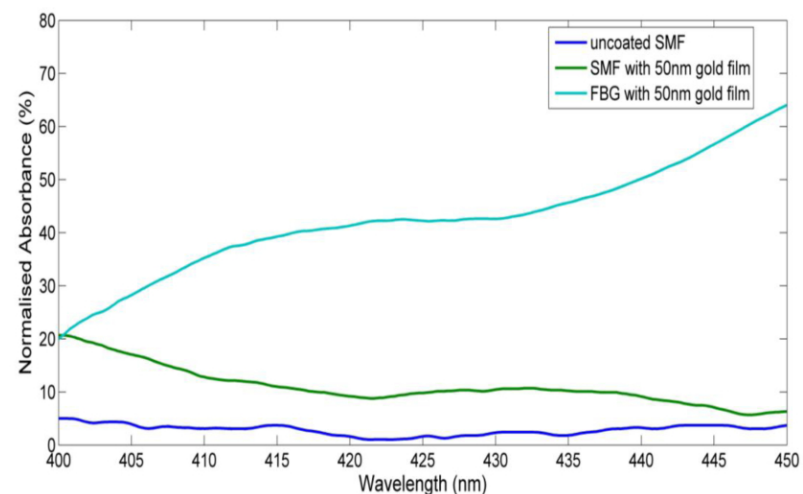

FIG. 3 Comparison of Absorbance Spectrum of SMF and FBG in air.

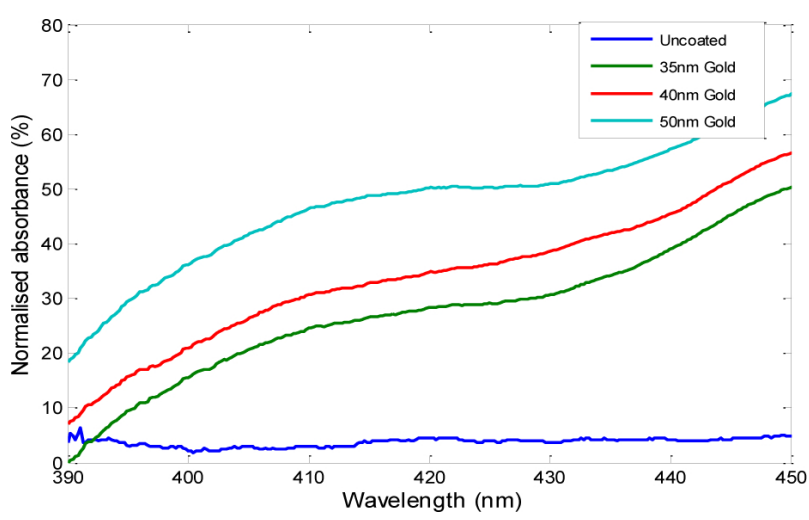

FIG. 4 Absorbance spectrum of uncoated and gold coated FBC sensor in air.

then to a computer which has Spectra Suite software for data processing.

Absolute ethanol with $99.7 \%$ purity was diluted with deionised water to produce various concentrations of ethanol for sensing purposes. The liquid is then deposited into the flow cell and the sensor output is observed. The absorbance readings from the spectrophotometer are recorded for different concentrations of ethanol in water.

The dynamic response of the sensor was also observed, by alternating two concentrations of ethanol, 5\% and 20\% in the flow cell. The average absorbance level at a wavelength range from $400 \mathrm{~nm}$ to $500 \mathrm{~nm}$ is plotted by the Spectra suite software in real time.

\section{RESULTS AND DISCUSSION}

The absorbance spectrum of a standard SMF and gold coated SMF was observed. The absorbance increases when a thin gold film is sputtered over a $1 \mathrm{~cm}$ area of an SMF where the buffer coating is removed. This result was compared with a gold coated FBG and a significant increase in absorbance was observed as shown in Figure 3. This is due to the Bragg scattering effect of the FBG which causes more light to be scattered to the cladding, resulting in higher absorption by the gold film.

Further investigations were carried out using FBGs coated with a layer of gold film. It was observed, as shown in Figure 4 , that the absorbance increases as the thickness of the gold layer was increased.

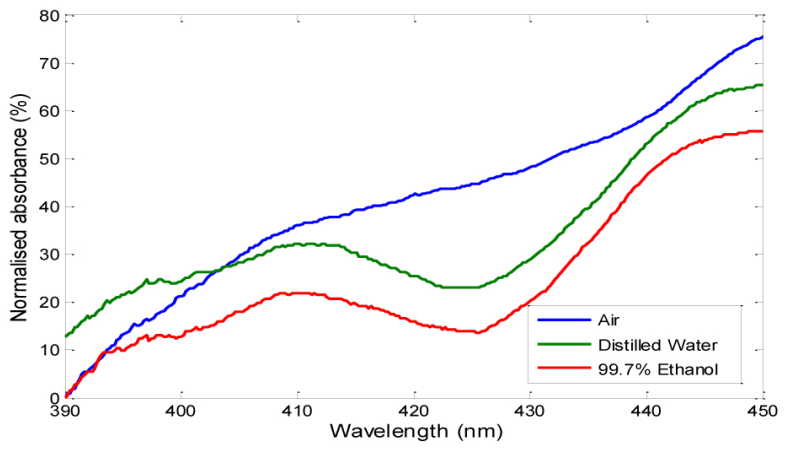

FIG. 5 Absorbance spectrum of $50 \mathrm{~nm}$ gold coated FBG sensor in various mediums.

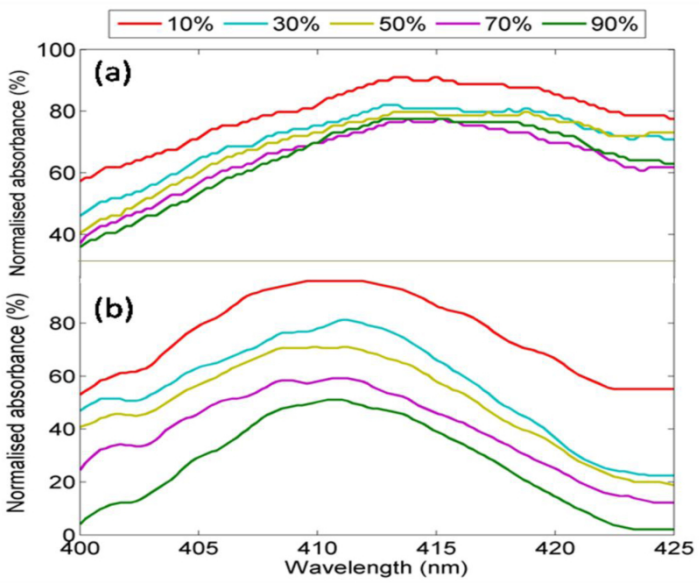

FIG. 6 Normalised Absorbance of (a) $35 \mathrm{~nm}$ and (b) $50 \mathrm{~nm}$ gold coated FBG sensor for different concentrations of ethanol in water.

The FBG sensor was then immersed in water and then in ethanol. A significant absorbance peak is observed at $410 \mathrm{~nm}$ followed by a dip at $425 \mathrm{~nm}$, which did not exist when the sensor was exposed to air as shown in Figure 5.

These changes in absorbance are caused by the $\mathrm{O}-\mathrm{H}$ stretching of the water molecules. This absorbance spectrum of the FBG sensor towards pure water corresponds to earlier studies by Pope et. al. [25].

Figure 6(a) and (b) show the normalised absorbance of $35 \mathrm{~nm}$ and $50 \mathrm{~nm}$ gold coated FBG sensors exposed to water with different ethanol concentrations. Two FBG sensors with gold film thickness of $35 \mathrm{~nm}$ and $50 \mathrm{~nm}$ were immersed into different concentrations of ethanol, ranging from $0 \%$ to $99.7 \%$ with increments of $10 \%$. These two gold film thicknesses were chosen due to previous work by Suzuki et. al. suggested that maximum evanescent field enhancement occurs at range from 30 to $50 \mathrm{~nm}$ [26]. It was observed that, in the visible region, the absorbance decreases when the ethanol concentration in water was increased which corresponds with the results in Figure 5 . This agrees well with the work reported by Xiong et. al. [14]. The increase in the absorbance levels may be contributed by the presence of the carbon molecules introduced during the addition of the ethanol, that interferes with the hydrogen bonding of the water molecules [27].

The average normalised absorbance results from $400 \mathrm{~nm}$ to $425 \mathrm{~nm}$ from Figure 6(a) and 6(b) are plotted in Figure 7. Lin- 


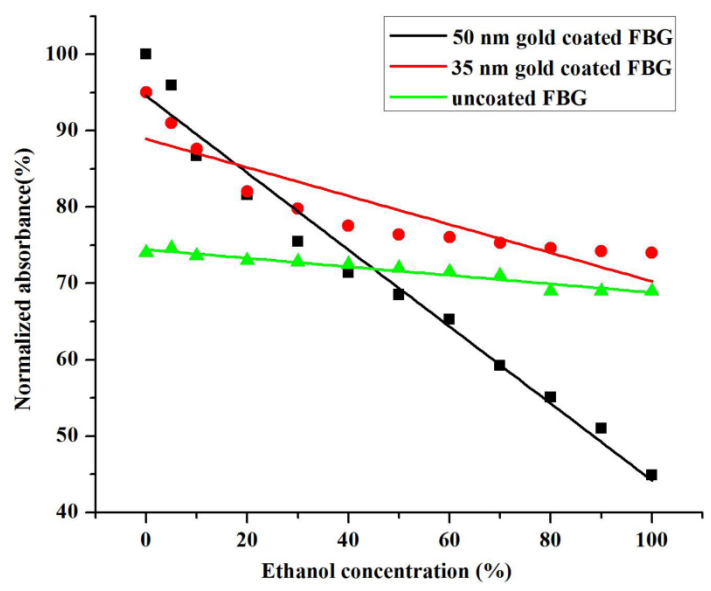

FIG. 7 Average normalised absorbance of FBG sensors with different gold thickness for various concentrations of ethanol in water.

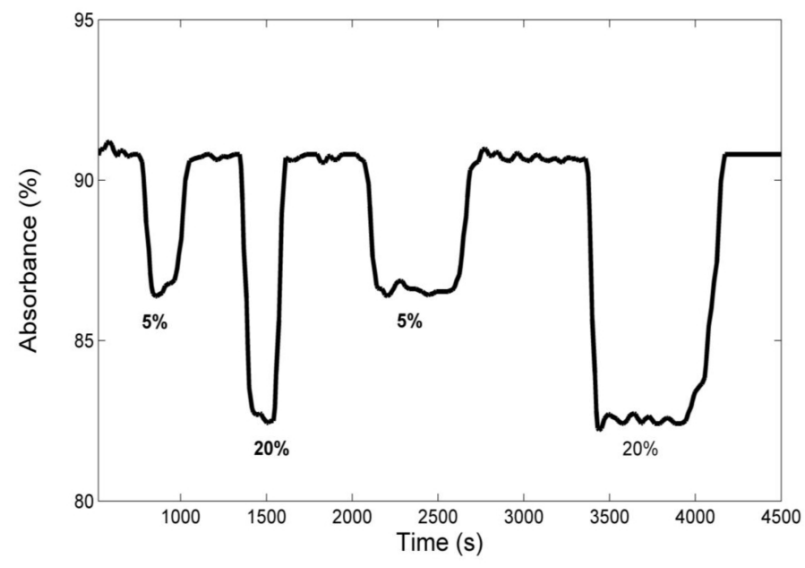

FIC. 8 Dynamic response of $50 \mathrm{~nm}$ gold coated FBC sensor towards various ethanol concentration.

ear fitting was performed on the data and the absorbance response show good linearity $0.96996,0.77189$ and 0.94138 for the $50 \mathrm{~nm}$ gold coated, $35 \mathrm{~nm}$ gold coated and uncoated FBG respectively. The slopes of the linear fitted graphs are -0.504 for the $50 \mathrm{~nm}$ gold coated FBG, -0.186 for the $35 \mathrm{~nm}$ gold coated FBG and -0.056 for the uncoated FBG. Based on these values, the $50 \mathrm{~nm}$ gold coated FBG offers the best sensitivity of the three sensors above. This could be attributed to the fact that the penetration length of the evanescent field for gold is optimum at $50 \mathrm{~nm}$ thickness, which allows a larger surface for the interaction of the evanescent wave with the surrounding medium [28].

Finally the dynamic response of the $50 \mathrm{~nm}$ gold coated FBG sensor was investigated as shown in Figure 8. The sensor offers excellent repeatability and sensitivity of approximately $2 \%$ change in absorbance for every $10 \%$ change in ethanol concentration. The developed sensor was also highly stable as indicated by the steady baseline when it was immersed in the ethanol for different time periods with different ethanol concentrations. The response and recovery times of the gold coated FBG sensor are approximately 20 and 35 seconds, respectively.

\section{CONCLUSION}

A fiber optic absorbance sensor was successfully developed to detect various concentrations of ethanol in water. Thin layer of gold film was sputtered over a fully cladded FBG to enhance the evanescent field interacting with the surrounding medium. The FBG sensor was tested for different concentrations of ethanol in water via spectroscopy. The sensor showed good results for both high and low concentrations of ethanol. Best results were obtained when sensor was sputtered with a gold film thickness of $50 \mathrm{~nm}$. The robust gold coated FBG sensor shows great potential for evanescent wave based sensors in volatile environments.

\section{ACKNOWLEDGEMENTS}

The work reported in this paper is partly supported by the Universiti Putra Malaysia's Research University Grant Schemes (Ref: 05-01-12-1626RU and 05-02-12-2015RU) and Ministry of Higher Education, Malaysia's Fundamental Research Grant Scheme (Ref: 03-04-10-795FR).

\section{References}

[1] M. Bravo, A. Candiani, A. Cucinotta, S. Selleri, M. Lopez-Amo, J. Kobelke, and K. Schuster, "Remote PCF-based sensors multiplexing by using optical add-drop multiplexers," Opt. Laser Technol. 57, 911 (2014).

[2] K. S. C. Kuang, S. T. Quek, and M. Maalej, "Remote flood monitoring system based on plastic optical fibres and wireless motes," Sensor. Actuat. A-Phys. 147(2), 449-455 (2008).

[3] M. Niggemann, A. Katerkamp, M. Pellmann, P. Bolsmann, J. Reinbold, and K. Cammann, "Remote sensing of tetrachloroethene with a micro-fibre optical gas sensor based on surface plasmon resonance spectroscopy," Sensor. Actuat. B-Chem. 34(1)-(3), 328333 (1996).

[4] A. K. Sharma and B. D. Gupta, "Theoretical model of a fiber optic remote sensor based on surface plasmon resonance for temperature detection," Opt. Fiber Technol. 12(1), 87-100 (2006).

[5] J. M. Bello, V. A. Narayanan, D. L. Stokes, and V. D. Tuan, “Fiberoptic remote sensor for in situ surface-enhanced Raman scattering analysis," Anal. Chem. 62(22), 2437-2441 (1990).

[6] Y. Zhao, and C. Zhou, "Fast characterization of low-reflectance Bragg gratings in a polarization maintaining fiber using a reference grating," Opt. Fiber Technol. 17(3), 242-246 (2011).

[7] G. Breglio, A. Irace, A. Cusano, and A. Cutolo, "Chirped-pulsed frequency modulation (C-PFM) for fiber Bragg grating sensors multiplexing," Opt. Fiber Technol. 12(1), 71-86 (2006).

[8] B. Jiang, J. Zhao, C. Qin, Z. Huang, and F. Fan, “An optimized strain demodulation method based on dynamic double matched fiber Bragg grating filtering," Opt. Laser. Eng. 49(3), 415-418 (2011).

[9] R. Ruzek, P. Kudrna, M. Kadlec, V. Karachalios, and K. I. Tserpes, "Strain and damage monitoring in CFRP fuselage panels using fiber Bragg grating sensors. Part II: Mechanical testing and validation," Compos. Struct. 107, 737-744 (2014).

[10] H. Peng, Y. Su, Z. Ye, and B. Zhou, “A novel fiber Bragg grating sensor for weak pressure measurement based on the Stokes parameter," Opt. Fiber Technol. 18(6), 485-489 (2012). 
[11] I. de Lourenço, G. R. C. Possetti, M. Muller, and J. L. Fabris, “Fiber Bragg grating sensor to monitor stress kinetics in drying process of commercial latex paints," Sensors 10, 4761-76 (2010).

[12] P. A. S. Jorge, S. O. Silva, C. Gouveia, P. Tafulo, L. Coelho, P. Caldas, D. Viegas, et al., "Fiber optic-based refractive index sensing at INESC Porto," Sensors 12(6), 8371-8389 (2012).

[13] J. Dai, M. Yang, X. Yu, K. Cao, and J. Liao, "Greatly etched fiber Bragg grating hydrogen sensor with $\mathrm{Pd} / \mathrm{Ni}$ composite film as sensing material," Sensor. Actuat. B-Chem. 174, 253-257 (2012).

[14] F. B. Xiong and D. Sisler, "Determination of low-level water content in ethanol by fiber-optic evanescent absorption sensor," 0 pt. Commun. 283(7), 1326-1330 (2010).

[15] J. Dai, M. Yang, X. Yu, and H. Lu, “Optical hydrogen sensor based on etched fiber Bragg grating sputtered with $\mathrm{Pd} / \mathrm{Ag}$ composite film," Opt. Fiber Technol. 19(1), 26-30 (2013).

[16] J. Dai, M. Yang, Z. Yang, Z. Li, Y. Wang, G. Wang, Y. Zhang, and $Z$. Zhuang, "Performance of fiber Bragg grating hydrogen sensor coated with Pt-loaded W03 coating," Sensor. Actuat. B-Chem. 190, 657-663 (2014).

[17] M. Kumar, D. Engles, S. Prashar and A. Singh, "Etched FBG as chemical sensor for fuel adultration," IJERT 1(4), 1-5 (2012).

[18] G. Laffont, and P. Ferdinand, "Tilted short-period fibre-Bragggrating-induced coupling to cladding modes for accurate refractometry," Meas. Sci. Technol. 12, 765-770 (2001).

[19] G. Nemova, and R. Kashyap, "Modeling of Plasmon-Polariton Refractive-Index Hollow Core Fiber Sensors Assisted by a Fiber Bragg Grating," J. Lightwave Technol. 24(10), 3789-3796 (2006).
[20] N. Díaz-herrera, D. Viegas, P. A. S. Jorge, F. M. Araújo, J. Santos, M. Navarrete, and A. Conzález-cano, "Chemical Fibre-optic SPR sensor with a FBC interrogation scheme for readout enhancement," Sensor. Actuat. B-Chem. 144, 226-231 (2010).

[21] Y. Y. Shevchenko, and J. Albert, "Plasmon resonances in goldcoated tilted fiber Bragg gratings.," Opt. Lett. 32(3), 211-213 (2007).

[22] J. Albert, L.-Y. Shao, and C. Caucheteur, "Tilted fiber Bragg grating sensors," Laser Photonics Rev. 7(1), 83-108 (2013).

[23] S. Capouilliet, J. A. Smith, D. J. Walter, G. S. Glaesemann, G. E. Kohnke, and R. D. Irion, "A Fiber Bragg Grating Measurement System for Monitoring Optical Fiber Strain," (2011), http://www.corning.com/docs/opticalfiber/tr3680.pdf.

[24] F. B. Xiong, W. Z. Zhu, H. F. Lin, and X. G. Meng, "Fiber-optic sensor based on evanescent wave absorbance around $2.7 \mu \mathrm{m}$ for determining water content in polar organic solvents," Appl. Phys. B 115, 129-135 (2013).

[25] R. M. Pope, and E. S. Fry, "Pure water II Integrating cavity measurements," Appl. Optics 36(33), 8710-8723 (1997).

[26] H. Suzuki, M. Sugimoto, Y. Matsui, and J. Kondoh, "Effects of gold film thickness on spectrum profile and sensitivity of a multimodeoptical-fiber SPR sensor," Sensor. Actuat. B-Chem. 132(1), 26-33 (2008).

[27] P. Zumbo, "Ethanol Precipitation," Pauling 1932, 1-12 (1932).

[28] B. D. Gupta, and R. K. Verma, "Surface Plasmon Resonance-Based Fiber Optic Sensors: Principle, Probe Designs, and Some Applications," Journal of Sensors 2009, 1-12 (2009). 